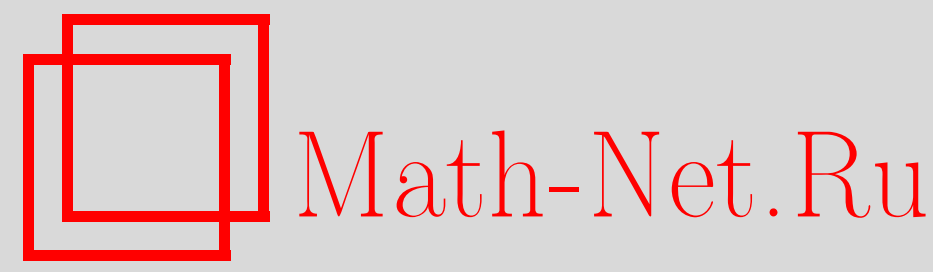

Р. С. Юлмухаметов, Целые функции многих переменных с заданным поведением в бесконечности, Изв. РАН. Сер. матем., 1996, том 60, выпуск 4, 205-224

DOI: https://doi.org/10.4213/im82

Использование Общероссийского математического портала Math-Net.Ru подразумевает, что вы прочитали и согласны с пользовательским соглашением http://www . mathnet.ru/rus/agreement

Параметры загрузки:

IP: 18.207 .199 .55

26 апреля 2023 г., 03:05:57 
УДК 517.574

\author{
Р.С. Юлмухаметов
}

\title{
Целые функции многих переменных с заданным поведением в бесконечности
}

\begin{abstract}
Доказывается теорема о возможности асимптотической аппроксимации плюрисубгармонической функции, имеющей конечный порядок роста, посредством логарифмирования модуля целой функции.

Библиография: 12 наименований.
\end{abstract}

\section{Введение}

В данной работе рассматривается вопрос об асимптотическом приближении заданной плюрисубгармонической функции $u(z)$ в $\mathbb{C}^{p}$ посредством логарифма модуля целой функции. Для случая одной переменной этот вопрос подробно изучен в работах [1]-[5], и в конечном итоге доказано утверждение: для любой субгармонической в $\mathbb{C}$ функции $u(z)$, удовлетворяющей условию

$$
u(z) \leqslant C \cdot(1+|z|)^{\rho}, \quad z \in \mathbb{C}, \quad C \text { - const },
$$

сушествует целая функция $f$ такая, что

$$
|u(z)-\ln | f(z)|| \leqslant C \cdot \ln (1+|z|), \quad z \notin E, \quad C \text { - const }
$$

причем исключительное множество $E$ может быть покрыто системой кругов с конечной суммой радиусов.

Для многих переменных общий результат об аппроксимации плюрисубгармонических функций конечного порядка роста $\rho$ был получен Р. Сигурдсоном [6] и, независимо, автором [7]. Оба эти результата опубликованы в препринтах и являются малодоступными. В связи с этим в данной статье мы излагаем упрошенный вариант доказательства основного результата из работы [7].

Обозначения: $D_{m}(x, r)$ - шар с центром в точке $x \in \mathbb{R}^{m}$ радиуса $r ; d \lambda_{m}$-мера Лебега в $\mathbb{R}^{m} ; \mu_{u}$-мера, ассоциированная по Риссу с субгармонической функцией $u$.

\section{§1. Приближение при дополнительных условиях}

Основным содержанием этого параграфа является доказательство следующей теоремы.

(C) Р.С. Юлмухаметов 1996 
Теорема 1. Пусть $\varphi$ - плюрисубгармоническая функиия в $\mathbb{C}^{p}$, удовлетворяющая условиям

$$
\begin{gathered}
\varphi(0)=0, \\
\varphi(z) \leqslant A(|z|+1)^{\rho}, \quad z \in \mathbb{C}^{p}, \\
|\varphi(z)-\varphi(w)| \leqslant B(1+|z|)^{\gamma-1}|z-w|, \quad \text { ecлu } \quad|z-w| \leqslant(1+|z|)^{1-\frac{\gamma}{3}} .
\end{gathered}
$$

Тогда существует целая функиия $F$ в $\mathbb{C}^{p}$, которая вне множества

$$
S_{F}(\alpha, d)=\bigcup_{F(z)=0} D_{2 p}\left(z, d(1+|z|)^{\alpha}\right)
$$

удовлетворяет соотношению

$$
|\varphi(z)-\ln | F(z)|| \leqslant C(|z|+1)^{\frac{2}{3} \gamma} \ln (|z|+e) .
$$

При этом постоянная $C$ зависит от постоянных $\rho, \gamma, A, B, \alpha, d, d>0$, но не зависит от конкретного вида функции $\varphi$.

В ходе доказательства этой теоремы нам потребуется следуюшее утверждение (см. $[8$, с. 132]).

Лемма 1. Пусть $\varphi-$ плюрисубгармоническая функиия в $\mathbb{C}^{p}$, удовлетворяющая условию

$$
|\varphi(z)-\varphi(w)| \leqslant M, \quad \text { еслu }|z-w| \leqslant(1+|z|)^{\gamma},
$$

где $\gamma<0$ - некоторое число. Пусть $\Sigma-$ комплексная плоскость в $\mathbb{C}^{p}$. Для всякой аналитической функции $f$ на $\Sigma$ такой, что

$$
\ln |f(z)| \leqslant \varphi(z)
$$

существует целая функция $F$ в $\mathbb{C}^{p}$ такая, что $F=f$ на $\Sigma и$

$$
\ln |F(z)| \leqslant \varphi(z)+N_{p} \ln \left(|z|+e+d_{\Sigma}\right), \quad z \in \mathbb{C}^{p},
$$

где $d_{\Sigma}$ - расстояние от $\Sigma$ до начала координат и постоянная $N_{p}$ зависит лишь от $M, \gamma$ и не зависит от функций $\varphi, f$ и от $\Sigma$.

ДоКАЗАТЕЛЬСтво. Можно считать, что $\Sigma$-гиперплоскость $z_{p}=a$. При этом $d_{\Sigma}=|a|$. Рассмотрим функцию

$$
\widetilde{\varphi}(z)=\varphi\left(z_{1}, \ldots, z_{p-1}, z_{p}+a\right) .
$$

В силу соотношения (4) с учетом отрицательности $\gamma$ функция $\widetilde{\varphi}$ удовлетворяет условию

$$
|\widetilde{\varphi}(z)-\widetilde{\varphi}(w)| \leqslant M, \text { если }|z-w| \leqslant(1+|z|+|a|)^{\gamma} .
$$

Функция

$$
\tilde{f}(z)=f\left(z_{1}, \ldots, z_{p-1}, z_{p}+a\right)
$$


определена и голоморфна на гиперплоскости $z_{p}=0$. Функция $\tilde{f}$ - голоморфная функция переменной $z^{\prime}=\left(z_{1}, \ldots, z_{p-1}\right)$, и можно рассматривать $\tilde{f}$ как голоморфную функцию в $\mathbb{C}^{p}$, не зависящую от $z_{p}$. Положим

$$
E=\left\{z=\left(z_{1}, \ldots, z_{p}\right) \in \mathbb{C}^{p}:\left|z_{p}\right| \leqslant\left(1+\left|z^{\prime}\right|+|a|\right)^{\gamma}\right\} .
$$

Согласно (5) и (6) выполняется неравенство

$$
\begin{aligned}
& \int_{E}|\tilde{f}|^{2} \exp \left\{-2 \widetilde{\varphi}(z)-2(p-\gamma) \ln \left(\left|z^{\prime}\right|+|a|+1\right)\right\} d \lambda_{2 p}(z) \\
& \quad \leqslant \int_{\mathbb{C}^{p-1}} \exp \left\{2 M-2(p-\gamma) \ln \left(\left|z^{\prime}\right|+|a|+1\right)\right\} \pi\left(1+\left|z^{\prime}\right|+|a|\right)^{2 \gamma} d \lambda_{2(p-1)}\left(z^{\prime}\right) \\
& \quad \leqslant \pi e^{2 M} \int_{\mathbb{C}^{p-1}}\left(1+\left|z^{\prime}\right|\right)^{-2 p} d \lambda_{2(p-1)}\left(z^{\prime}\right) \leqslant \pi c_{p-1} e^{2 M},
\end{aligned}
$$

где $c_{p-1}$ - площадь единичной сферы в $\mathbb{C}^{p-1}$. Пусть $\psi(z)=\psi(|z|)$ - гладкая функция в $C$, равная 1 в круге радиуса $1 / 2$ и 0 вне единичного круга с центром в нуле. Тогда

$$
\left|\bar{\partial}\left(\psi\left(z_{p}\left(1+\left|z^{\prime}\right|+|a|\right)^{-\gamma}\right)\right)\right| \leqslant(1+(p-1)|\gamma|) M_{0}\left(1+\left|z^{\prime}\right|+|a|\right)^{-1},
$$

где

$$
M_{0}=\max _{r>0}\left|\psi^{\prime}(r)\right|
$$

Полагая

$$
\widetilde{F}(w)=\psi\left(w_{p}\left(1+\left|w^{\prime}\right|+|a|\right)^{-\gamma}\right) \tilde{f}(w)-w_{p} v(w),
$$

получаем, что $\widetilde{F}(w)=\tilde{f}(w)$, когда $w_{p}=0$; значит, остается только выбрать $v$ так, чтобы она удовлетворяла подходящей оценке и чтобы $\bar{\partial} \widetilde{F}=0$, т.е.

$$
\bar{\partial} v=z_{p}^{-1} \tilde{f}\left(z^{\prime}\right) \bar{\partial} \psi\left(z_{p}\left(1+\left|z^{\prime}\right|+|a|\right)^{-\gamma}\right)=g .
$$

Ясно, что $\bar{\partial} g=0$. Учитывая, что

$$
\bar{\partial} \psi\left(z_{p}\left(1+\left|z^{\prime}\right|+|a|\right)^{-\gamma}\right)=0
$$

при

$$
\left|z_{p}\right|<\frac{1}{2}\left(1+\left|z^{\prime}\right|+|a|\right)^{\gamma}
$$

и $z \notin E$, мы из (7) получим

$$
\begin{aligned}
& \int|g|^{2} \exp \left\{-2 \widetilde{\varphi}(z)-2(p-2 \gamma+1) \ln \left(1+\left|z^{\prime}\right|+|a|\right)\right\} d \lambda_{2 p}(z) \\
& \leqslant 4 \pi e^{2 M}(1+(p-1)|\gamma|)^{2} M_{0}^{2} c_{p-1}=\sigma .
\end{aligned}
$$

Теперь теорема Л. Хермандера (см. [8, с. 132]) дает решение уравнения $\bar{\partial} v=g$, для которого справедлива оценка

$$
\int \frac{|v|^{2}}{\left(1+|z|^{2}\right)^{2}} \exp \left\{-2 \widetilde{\varphi}(z)-2(p-2 \gamma+1) \ln \left(1+\left|z^{\prime}\right|+|a|\right)\right\} d \lambda_{2 p}(z) \leqslant \sigma .
$$


Комбинируя эту оценку с оценкой для интеграла от $\tilde{f}$, получим

$$
\int \frac{|\widetilde{F}|^{2}}{\left(1+|z|^{2}\right)^{2}} \exp \left\{-2 \widetilde{\varphi}(z)-2(p-2 \gamma+1) \ln \left(1+\left|z^{\prime}\right|+|a|\right)\right\} d \lambda_{2 p}(z) \leqslant \sigma_{1} .
$$

Функция

$$
F(z)=\widetilde{F}\left(z_{1}, \ldots, z_{p-1}, z_{p}-a\right)
$$

голоморфна в $\mathbb{C}^{p}$, равна $f$ на гиперплоскости $\Sigma$ и удовлетворяет соотношению

$$
\int \frac{|F(z)|^{2}}{(1+|z|+|a|)^{4}} \exp \left\{-2 \varphi(z)-2(p-2 \gamma+1) \ln \left(1+\left|z^{\prime}\right|+|a|\right)\right\} d \lambda_{2 p}(z) \leqslant \sigma_{1} .
$$

Отсюда и из неравенства

$$
|F(z)|^{2} \leqslant \frac{1}{c_{p} r^{2 p}} \int_{|w| \leqslant r}|F(z+w)|^{2} d \lambda_{2 p}(w)
$$

с учетом условия (4) получим утверждение леммы 1.

Докажем теорему 1 при $p=1$ в более сильном варианте.

Теорема $1^{\prime}$. Пусть $\varphi$-субгармоническая функция, удовлетворяющая условиям⿻

$$
\begin{gathered}
\varphi(0)=0 \\
\varphi(z) \leqslant A^{\prime}(1+|z|+R)^{\rho} \quad \text { для некоторого } \quad R>0 \\
|\varphi(z)-\varphi(w)| \leqslant B^{\prime}(1+|z|+R)^{\beta}|z-w|, \quad \text { если } \quad|z-w| \leqslant(1+|z|+R)^{-\beta} .
\end{gathered}
$$

Тогда существует челая функиия $F$, которая вне множества

$$
S_{F}(\alpha, d, R)=\bigcup_{F(z)=0} D\left(z, d(1+|z|+R)^{\alpha}\right)
$$

удовлетворяет соотношению

$$
|\varphi(z)-\ln | F(z)|| \leqslant C \cdot \ln (|z|+R+e) .
$$

Верхняя оченка

$$
\ln |F(z)| \leqslant \varphi(z)+C \cdot \ln (|z|+R+e)
$$

выполняется всюду в $\mathbb{C}$. При әтом константа $C$ зависит от параметров $A^{\prime}, B^{\prime}, \alpha, \beta, d, d>0$, но не зависит от функции $\varphi$ и от параметра $R$. 
ДокАЗАТЕЛЬСТВо проведем на основании результатов работы [4]. Для неотрицательной борелевской меры $\mu$ через $\mu(z, t)$ обозначим $\mu$-меру шара $D(z, t)$. Точку $z$ назовем $(b, s)$-нормальной по мере $\mu$, если выполнено условие

$$
\mu(z, t) \leqslant b t, \quad t \in(0, s) .
$$

Из формулы Привалова и из условия $\left(3^{\prime}\right)$ следует оценка для ассоциированной меры $\mu_{\varphi}$ функции $\varphi$ :

$$
\int_{0}^{t} \frac{\mu_{\varphi}(z, \tau)}{\tau} d \tau=\frac{1}{2 \pi} \int_{0}^{2 \pi} \varphi\left(z+t e^{i \theta}\right) d \theta-\varphi(z) \leqslant B^{\prime}(1+|z|+R)^{\beta} t
$$

когда $t \leqslant(1+|z|+R)^{-\beta}$. Так как $\mu(z, t)-$ неубывающая функция переменной $t$, то имеем

$$
\mu_{\varphi}(z, t) \leqslant \int_{t}^{e t} \frac{\mu_{\varphi}(z, \tau)}{\tau} d \tau \leqslant B^{\prime} e(1+|z|+R)^{\beta} t,
$$

когда $t \leqslant e^{-1}(1+|z|+R)^{-\beta}$, т.е. точка $z$ является $\left(B^{\prime} e(1+|z|+R)^{\beta}, e^{-1}(1+\right.$ $\left.|z|+R)^{-\beta}\right)$-нормальной по мере $\mu_{\varphi}$.

Применим к функции $\varphi$ теорему $4^{\prime}$, затем теорему 4 из работы [4]. Из этих теорем вытекает существование целой функции $F(z)$, которая в точках, $(b, s)$-нормальных по мерам $\mu_{\varphi}$ и $\mu_{\ln |F|}$, удовлетворяет оценке

$$
|\varphi(z)-\ln | F(z)|| \leqslant A_{0}|\ln s|+B_{0} \ln (|z|+1)+b s(|\ln s|+1)+C_{0},
$$

где постоянные $A_{0}, B_{0}, C_{0}$ не зависят от функции $\varphi$.

Существует число $l>0$ такое, что точка $z \notin S_{F}(\alpha, d, R)$ является $(0, l(1+$ $\left.|z|+R)^{\alpha}\right)$-нормальной по мере $\mu_{\ln |F|}$. Если в соотношении (9) положим

$$
\begin{aligned}
& b=e B^{\prime}(1+|z|+R)^{\beta}, \\
& s=\min \left(e^{-1}(1+|z|+R)^{-\beta}, l(1+|z|+R)^{\alpha}\right),
\end{aligned}
$$

то получим оценку (8). Верхняя оценка следует из замечания к лемме 3.1 из работы [4].

Следуюшая лемма непосредственно вытекает из теоремы 6 из работы [4].

ЛЕмма 2. Для любого $\gamma>0$ существует челая функция $g(z)$ в $\mathbb{C}$, удовлетворяющая условиям:

1) все нули $\left(a_{n}\right)_{n=1}^{\infty}$ функиии $g$ простые и при некоторых $d>0$ и $\alpha$ круги $D_{2}\left(a_{n}, d\left(1+\left|a_{n}\right|\right)^{\alpha}\right)$ попарно не пересекаются;

2) вне кругов $D_{2}\left(a_{n}, d\left(1+\left|a_{n}\right|\right)^{\alpha}\right)$ выполняется соотношение

$$
\left.|\ln | g(z)|-| z\right|^{\gamma} \mid \leqslant C \cdot \ln (|z|+e), \quad C \text { - const }
$$

причем верхняя оченка

$$
\ln |g(z)| \leqslant|z|^{\gamma}+\cdot \ln (|z|+e), \quad C-\text { const },
$$


выполняется на всей плоскости.

Заметим, что из формулы Коши

$$
\frac{1}{g^{\prime}\left(a_{n}\right)}=\frac{1}{2 \pi i} \int_{\left|z-a_{n}\right|=d\left(1+\left|a_{n}\right|\right)^{\alpha}} \frac{d \zeta}{g(z)}
$$

вытекает оценка

$$
\left|g^{\prime}\left(a_{n}\right)\right| \geqslant d_{1} \exp \left\{\left|a_{n}\right|^{\gamma}-C \cdot \ln \left(\left|a_{n}\right|+e\right)\right\}, \quad C \text { - const. }
$$

Кроме того, можно считать, что для некоторого $x>0$ имеет место равенство

$$
\bigcup_{n=1}^{\infty} D_{2}\left(a_{n}, x\left(1+\left|a_{n}\right|\right)^{1-\frac{\gamma}{3}}\right)=\mathbb{C} .
$$

Для положительного $\rho$ через $K_{\rho}$ обозначим класс положительных монотонных функций $q(x)$ на $\mathbb{R}^{+}$, удовлетворяюших условиям

$$
\begin{aligned}
q(t x) & \leqslant t^{\rho} q(x), \quad t \geqslant 1, \quad x>0, \\
q(x+1) & \leqslant 2^{\rho} q(x), \quad x \in(0,1) .
\end{aligned}
$$

Лемма 3. Существуют постоянные $A, B>1$ такие, что для любой функчии $q \in K_{\rho}$ и для любой субгармонической функиии $\varphi$, удовлетворяющей условиям

(a) $\varphi(0)=0$,

(b) $\varphi(z) \leqslant q(|z|), \quad z \in \mathbb{C}$,

в точках $z,(\beta, s)$-нормальных по мере $\mu_{\varphi}$, выполняется оченка снизу

$$
\varphi(z) \geqslant-A B^{\rho} q(|z|)\left[\ln +\frac{3|z|}{s}+1\right]-\beta s .
$$

ДокаЗАтЕльство. Воспользуемся представлением Рисса в круге $D_{2}(0,2|z|)$ :

$$
\varphi(z)=H(z)-\int G(z, w) d \mu_{\varphi}(w) .
$$

Используя элементарные оценки ядра Пуассона и условие (b), получим

$$
\begin{aligned}
H(z) & \geqslant \frac{1}{2 \pi} \int_{0}^{2 \pi} \frac{4|z|^{2}-|z|^{2}}{|z-2| z\left|e^{i \theta}\right|^{2}}\left(\varphi\left(2|z| e^{i \theta}\right)-\varphi^{+}\left(2|z| e^{i \theta}\right)\right) d \theta \\
& \geqslant \frac{3}{2 \pi} \int_{0}^{2 \pi} \varphi\left(2|z| e^{i \theta}\right) d \theta-3 q(2|z|) .
\end{aligned}
$$

Отсюда и из условия (a) вытекает оценка

$$
H(z) \geqslant-32^{\rho} q(|z|) .
$$


В силу формулы Привалова и условий (a), (b) имеем оценку

$$
\mu_{\varphi}(0, t) \leqslant q(e t) \leqslant e^{\rho} q(t), \quad t>0 .
$$

Чтобы оценить потенциал Грина, заметим, что ядро Грина области увеличивается с увеличением области. Если $G_{0}(z, w)$ - ядро Грина круга $D_{2}(z, 3|z|)$, то для любых $\zeta, w \in D_{2}(0,2|z|)$ выполняется неравенство

$$
G(\zeta, w) \leqslant G_{0}(\zeta, w)
$$

Применим это неравенство в точке $\zeta=z$ :

$$
\begin{aligned}
\int G(z, w) d \mu_{\varphi}(w) & \leqslant \int_{D_{2}(0,2|z|)} G_{0}(z, w) d \mu_{\varphi}(w) \\
& \leqslant \int_{D_{2}(0,2|z|)} \ln \frac{3|z|}{|z-w|} d \mu_{\varphi}(w)
\end{aligned}
$$

Таким образом, имеем оценку

$$
\int G(z, w) d \mu_{\varphi}(w) \leqslant \int_{0}^{3|z|} \ln \frac{3|z|}{t} d \tilde{\mu}(z, t)=\int_{0}^{3|z|} \frac{\tilde{\mu}(z, t)}{t} d t
$$

где через $\tilde{\mu}$ обозначено сужение меры $\mu_{\varphi}$ на круг $D_{2}(0,2|z|)$. Пользуясь $(\beta, s)$-нормальностью точки $z$ по мере $\tilde{\mu}$, получим

$$
\int G(z, w) d \mu_{\varphi}(w) \leqslant \beta s+\mu_{\varphi}(0,2|z|) \ln ^{+} \frac{3|z|}{s} .
$$

Вместе с оценкой гармонической мажоранты и величины $\mu(0,2|z|)$ это неравенство дает утверждение леммы 3.

ЛЕмма 4. Пусть $\varphi-$ плюрисубгармоническая функция, удовлетворяющая условиям (1), (2) и (3') при $R=0$. Тогда всюду в $\mathbb{C}^{p}$ имеет место неравенство

$$
\varphi(z) \geqslant-C \cdot(1+|z|)^{\rho} \ln (|z|+e),
$$

где константа $C$ зависит от $A, B^{\prime}, \rho, \beta$, но не зависит от функиии $\varphi$.

ДокАЗАТЕльСТво. Сужение функции $\varphi$ на любую плоскость, проходящую через точку $z=0$, удовлетворяет условиям леммы 3 для $q(x)=A(x+1)^{\rho}$. Причем в силу условия $\left(3^{\prime}\right)$ (при $\left.R=0\right)$ все точки $z$ плоскости являются $\left(B^{\prime} e(1+\right.$ $\left.|z|)^{\beta}, e^{-1}(1+|z|)^{-\beta}\right)$-нормальными по ассоциированной мере сужения. Применив лемму 3 к каждому сужению, получим утверждение леммы 4.

Изложим конструкцию функции $F$, удовлетворяюшей условиям теоремы 1 . По лемме 2 построим функцию $g$, удовлетворяющую соотношению

$$
\left.|\ln | g(z)|-| z\right|^{\frac{2}{3} \gamma} \mid \leqslant C \cdot \ln (|z|+e), \quad C \text { - const. }
$$


Пусть $S$ - множество нулей функции $g$. Через $B_{i}, i=1, \ldots, p$, обозначим $(p \times p)$-матрицу, у которой все элементы $i$-го столбца равны 1 , все элементы на главной диагонали, кроме $i$-го, равны $1 / 2$, а все остальные равны 0 . Через $b_{i j}$ обозначим $j$-ю вектор-строку матрицы $B_{i}$. При фиксированных $i$ и $\zeta_{j} \in S, j \neq i$, через $\Sigma_{\zeta}^{i}$ обозначим плоскость, задаваемую уравнением

$$
\left(z, b_{i j}\right)=\zeta_{j}, \quad j=1,2, \ldots, p, \quad j \neq i .
$$

На плоскости $\Sigma_{\zeta}^{i}$ введем параметризацию

$$
w(\xi)=z_{0}+\xi w_{0}, \quad\left|w_{0}\right|=1, \quad\left(z_{0}, w_{0}\right)=0 .
$$

Рассмотрим функцию

$$
\varphi_{\zeta, i}(\xi)=\varphi(w(\xi))-\varphi\left(z_{0}\right)
$$

Функция $\varphi_{\zeta, i}$ удовлетворяет условиям теоремы $1^{\prime}$ при $R=\left|z_{0}\right|$. В самом деле, условие $\left(1^{\prime}\right)$ очевидно. По условию (2) теоремы 1 имеем оценку

$$
\varphi(w(\xi)) \leqslant A(1+|w(\xi)|)^{\rho} \leqslant A\left(1+|\xi|+\left|z_{0}\right|\right)^{\rho} .
$$

Из условия (3) теоремы 1 следует, что функция $\varphi$ удовлетворяет условиям леммы 4 с $\beta=\gamma-1$, поэтому по лемме 4 имеем оценку

$$
\varphi\left(z_{0}\right) \geqslant-C \cdot\left(1+\left|z_{0}\right|\right)^{\rho} \ln \left(\left|z_{0}\right|+e\right),
$$

где константа $C$ зависит от постоянных $A, B, \rho$ и $\gamma$. Из последних двух оценок получим условие $\left(2^{\prime}\right)$ теоремы $1^{\prime}$ :

$$
\varphi_{\zeta, i}(\xi) \leqslant A^{\prime}\left(1+|\xi|+\left|z_{0}\right|\right)^{\rho+1} .
$$

Условие $\left(3^{\prime}\right)$ с $\beta=\gamma-1$ вытекает из условия (3). По теореме $1^{\prime}$ для каждого $\zeta=\left(\zeta_{j}\right), \zeta_{j} \in S, j \neq i$, сушествует целая функция $\tilde{f}_{\zeta, i}$ в $\mathbb{C}$, которая вне множества

$$
\widetilde{S}_{i}(\alpha, d, \zeta)=\bigcup_{\tilde{f}_{\zeta, i}(\xi)=0} D\left(\xi, d\left(1+\left|z_{0}\right|+|\xi|\right)^{\alpha}\right)
$$

удовлетворяет соотношению

$$
\left|\varphi_{\zeta, i}(\xi)-\ln \right| \tilde{f}_{\zeta, i}(\xi)|| \leqslant C \cdot \ln \left(|\xi|+\left|z_{0}\right|+e\right),
$$

и оценка сверху

$$
\ln \left|\tilde{f}_{\zeta, i}(\xi)\right| \leqslant \varphi_{\zeta, i}(\xi)+C \cdot \ln \left(|\xi|+\left|z_{0}\right|+e\right)
$$

выполняется для всех $\xi \in \mathbb{C}$. Причем, постоянная $C$ не зависит от $\zeta, i$.

Очевидно, функция $f_{\zeta, i}(w)=\tilde{f}_{\zeta, i}(\xi(w)) e^{\varphi\left(z_{0}\right)}, w \in \Sigma_{\zeta}^{i}$, удовлетворяет неравенствам

$$
\begin{gathered}
|\varphi(w)-\ln | f_{\zeta, i}(w)|| \leqslant C \ln (|w|+e), \quad w \notin \bigcup_{f_{\zeta, i}(z)=0} D_{2}\left(z, d(1+|z|)^{\alpha}\right), \\
\ln \left|f_{\zeta, i}(w)\right| \leqslant \varphi(w)+C \ln (|w|+e), \quad w \in \Sigma_{\zeta}^{i} .
\end{gathered}
$$


Пусть $d_{\zeta, i}-$ расстояние от начала координат до плоскости $\Sigma_{\zeta, i}$. По лемме 1 функция $f_{\zeta, i}$ может быть продолжена с плоскости $\Sigma_{\zeta}^{i}$ до функции $F_{\zeta, i}$, голоморфной на всем $\mathbb{C}^{p}$ и имеющей оценку

$$
\ln \left|F_{\zeta, i}(w)\right| \leqslant \varphi(w)+C \cdot \ln \left(|w|+e+d_{\zeta, i}\right) ;
$$

при этом постоянная $C$ не зависит от $\zeta, i$. Учитывая, что точка $z=\left(z_{1}, \ldots, z_{p}\right) \in$ $\mathbb{C}^{p}, z_{i}=0, z_{j}=2 \zeta_{j}, j \neq i$, лежит на плоскости $\Sigma_{\zeta}^{i}, \zeta=\left(\zeta_{1}, \ldots, \zeta_{i-1}, \zeta_{i+1}, \ldots, \zeta_{p}\right)$, получим

$$
d_{\zeta, i} \leqslant 2|\zeta| \leqslant 2\left(\left|\zeta_{1}\right|+\cdots+\left|\zeta_{p}\right|\right) .
$$

Поэтому для продолженной функции $F_{\zeta, i}$ верна оценка сверху

$$
\ln \left|F_{\zeta, i}(w)\right| \leqslant \varphi(w)+C\left(\ln (|w|+e)+\sum_{j \neq i} \ln \left(1+\left|\zeta_{j}\right|\right)\right) .
$$

При этом постоянная $C$ не зависит от $\zeta \in S^{p-1}$ и $i$.

Рассмотрим функцию

$$
\Phi_{i}(w)=\left(\prod_{k \neq i} g\left(\left(b_{k i}, w\right)\right)\right) \sum_{\zeta \in S^{p-1}} F_{\zeta, i}(w) \prod_{j \neq i} \frac{g\left(\left(w, b_{i j}\right)\right)}{g^{\prime}\left(\zeta_{j}\right)\left(\left(w, b_{i j}\right)-\zeta_{j}\right)}
$$

В силу соотношений $(12),(15)$ функция $\Phi_{i}$ целая и удовлетворяет верхней оценке

$$
\ln |\Phi(w)| \leqslant \varphi(w)+C \cdot(1+|w|)^{\frac{2}{3} \gamma}, \quad w \in \mathbb{C}^{p} .
$$

Кроме того, если $w \in \Sigma_{\zeta}^{l}$ при некотором $l \neq i$ и $\zeta=\left(\zeta_{1}, \ldots, \zeta_{l-1}, \zeta_{l+1}, \ldots, \zeta_{p}\right) \in$ $S^{p-1}$, то

$$
\Phi_{i}(w)=0 .
$$

Если $w \in \Sigma_{\zeta}^{i}$, то

$$
\Phi_{i}(w)=\left(\prod_{k \neq i} g\left(\frac{1}{2} w_{i}+w_{k}\right)\right) f_{\zeta, i}
$$

Отсюда в силу (13) и (12) получим, что для $w \in \Sigma_{\zeta}^{i} \backslash S_{\Phi_{i}}(\alpha, d)$ выполняется соотношение

$$
|\ln | \Phi_{i}(w)|-\varphi(w)| \leqslant C \cdot(1+|w|)^{\frac{2}{3} \gamma}, \quad C-\text { const. }
$$

Положим

$$
F(w)=\Phi_{1}(w)+\Phi_{2}(w)+\cdots+\Phi_{p}(w)
$$

Тогда целая функция $F(w)$ удовлетворяет верхней оценке

$$
\ln |F(w)| \leqslant \varphi(w)+C \cdot(1+|w|)^{\frac{2}{3} \gamma}, \quad C \text { - const, } \quad w \in \mathbb{C}^{p},
$$

и на плоскостях $\Sigma_{\zeta}^{i}$ вне множества $S_{F}(\alpha, d)$ имеет место соотношение

$$
|\ln | F(w)|-\varphi(w)| \leqslant \cdot(1+|w|)^{\frac{2}{3} \gamma}, \quad C \text { - const. }
$$

Воспользуемся следующей леммой. 
ЛЕмма 5. Пусть $f(z)$ - голоморфная функция в круге $D_{2}(0,2 e t), f(0)=1$. Положим $M=\max |f(\zeta)|$. Тогда в круге $D_{2}(0, t)$, но вне множества

$$
S_{f}(r)=\bigcup_{f(z)=0} D_{2}(z, r)
$$

верна оценка

$$
\ln |f(z)| \geqslant-\left(\ln \frac{3 e^{2} t}{r}\right) \ln M
$$

ДокАЗАТЕЛЬСТво. Построим функцию

$$
v(z)=\frac{(-2 t)^{n}}{a_{1} a_{2} \ldots a_{n}} \prod_{k=1}^{n} \frac{2 t\left(z-a_{k}\right)}{(2 t)^{2}-\bar{a}_{k} z}
$$

где $a_{1}, a_{2}, \ldots, a_{n}$ - корни функции $f$ в круге $D_{2}(0,2 t)$. Имеем $v(0)=1$ и $\left|v\left(2 t e^{i \theta}\right)\right|=$ $\frac{(2 t)^{n}}{\left|a_{1} a_{2} \ldots a_{n}\right|}$. Функция $\psi(z)=\frac{f(z)}{v(z)}$ не имеет нулей в круге $D_{2}(0,2 t)$, и по лемме Гельмгольца (см. $\left[12\right.$, с. 55]) в круге $D_{2}(0, t)$ выполняется нижняя оценка

$$
\ln |\psi(z)| \geqslant-2 \ln M+2 \ln \frac{(2 t)^{n}}{\left|a_{1} a_{2} \ldots a_{n}\right|} \geqslant-2 \ln M
$$

Оценим $\ln |v(z)|$. При $z \in D_{2}(0, t)$ имеем

$$
\prod_{k}\left|4 t^{2}-\bar{a}_{k} z\right| \leqslant\left(6 t^{2}\right)^{n}
$$

Если $z \in D_{2}(0, t) \backslash S_{f}(r)$, то

$$
\ln \prod_{k=1}^{n}\left|z-a_{k}\right| \geqslant n(2 t) \ln r
$$

где $n(\tau)$ обозначает число нулей функции $f$ в круге $D_{2}(0, \tau)$. Из последних двух неравенств вытекает, что

$$
\ln |v(z)| \geqslant-n(2 t) \ln \frac{3 t}{r} .
$$

Воспользовавшись неравенством $n(2 t) \leqslant \ln M$, которое следует из формулы Привалова, имеем при $z \in D(0, t) \backslash S_{f}(r)$

$$
\ln |v(z)| \geqslant-\ln \frac{(3 t)}{r} \ln M
$$

Вместе с (18) это неравенство доказывает лемму 5. 
Лемма 6. Пусть в точке $w_{0} \in \mathbb{C}^{p}$ выполняется соотношение (17), $\zeta_{0} \in \mathbb{C}^{p}$, $\left|\zeta_{0}\right|=1 u$

$$
f(z)=\frac{F\left(w_{0}+\zeta_{0} z\right)}{F\left(w_{0}\right)} .
$$

Тогда в круге $D_{2}\left(0, y\left(1+\left|w_{0}\right|\right)^{1-\frac{\gamma}{3}}\right)$, но для z таких, что $w_{0}+\zeta_{0} z \notin S_{F}(\alpha, d)$, выполняется неравенство

$$
\ln |f(z)| \geqslant-K(y)\left(1+\left|w_{0}\right|\right)^{\frac{2}{3} \gamma} \ln \left(\left|w_{0}\right|+e\right),
$$

где постоянная $K(y)$ не зависит от $w_{0}, \zeta_{0}$.

ДоКАЗАТЕЛЬСТВО. В силу условия (3) при $z \in D_{2}(0,2 e t), t=y\left(1+\left|w_{0}\right|\right)^{1-\frac{\gamma}{3}}$, учитывая неравенства (16), (17), имеем оценку

$$
\ln |f(z)| \leqslant C \cdot\left(1+\left|w_{0}\right|\right)^{\frac{2}{3} \gamma}, \quad C-\text { const. }
$$

Положим

$$
r=\min _{w \in D_{2}\left(w_{0}, 2 e t\right)} \min \left((1+|w|)^{1-\frac{\gamma}{3}}, d(1+|w|)^{\alpha}\right) .
$$

Заметим, что

$$
\ln \frac{t}{r} \leqslant C \cdot \ln \left(\left|w_{0}\right|+e\right), \quad C \text { - const. }
$$

По лемме 5 получим, что вне множества $S_{f}(r)$ выполняется оценка

$$
\ln |f(z)| \geqslant-C \cdot\left(1+\left|w_{0}\right|\right)^{\frac{2}{3} \gamma} \ln \left(\left|w_{0}\right|+e\right), \quad C \text { - const. }
$$

Число $r$ подобрано так, что если $z \in S_{f}(r)$, то $w_{0}+z \zeta_{0} \in S_{F}(\alpha, d)$. Поэтому в шаре $D_{2 p}\left(w_{0}, y\left(1+\left|w_{0}\right|\right)^{1-\frac{\gamma}{3}}\right)$, за исключением множества $S_{F}(\alpha, d)$, выполняется оценка

$$
\ln |F(w)| \geqslant \ln \left|F\left(w_{0}\right)\right|-C \cdot\left(1+\left|w_{0}\right|\right)^{\frac{2}{3} \gamma} \ln \left(\left|w_{0}\right|+e\right), \quad C \text { - const. }
$$

Снова используя условие (3), отсюда получим соотношение

$$
\begin{gathered}
|\ln | F(w)|-\varphi(w)| \leqslant C \cdot(1+|w|)^{\frac{2}{3} \gamma} \ln (|w|+e), \\
w \in D_{2 p}\left(w_{0},\left(1+\left|w_{0}\right|\right) y^{1-\frac{\gamma}{3}}\right) \backslash S_{F}(\alpha, d),
\end{gathered}
$$

где константа $C$ зависит от $y>0$ и не зависит от $w_{0}$.

Чтобы закончить доказательство теоремы 1 , покажем, что объединение шаров $D_{2 p}\left(w, y(1+|w|)^{1-\frac{\gamma}{3}}\right)$, где $w$ пробегает плоскости $\Sigma_{\zeta}^{i}, \zeta \in S^{p-1}, i=1, \ldots, p$, за исключением множеств $S_{i}(\alpha, d, \zeta)$, при некотором $y>0$ покрывает все пространство $\mathbb{C}^{p}$.

Для определенности будем считать, что $1-\frac{\gamma}{3}<0$. Пусть $w \in \mathbb{C}^{p}$ и $\left|w_{i}\right|=$ $\max _{j}\left|w_{j}\right|$. Тогда $\left|w_{i}\right|>\frac{1}{\sqrt{p}}|w|$. По соотношению (11) существуют $\zeta_{j} \in S, j \neq i$, такие, что

$$
\left|\left(w, b_{i j}\right)-\zeta_{j}\right| \leqslant x\left(1+\left|\zeta_{j}\right|\right)^{1-\frac{\gamma}{3}}
$$


где $x$ зависит от типа функции $g$. Поскольку

$$
\left|\left(w, b_{i j}\right)\right|=\left|w_{j}+\frac{1}{2} w_{j}\right| \geqslant \frac{1}{2 \sqrt{p}}|w|
$$

то из (19) получим

$$
\left(1+\left|\zeta_{j}\right|\right) \geqslant \frac{1}{4 \sqrt{p}}(1+|w|)
$$

Пусть

$$
w^{\prime}=\left(w_{1}^{\prime}, \ldots, w_{p}^{\prime}\right), \quad w_{i}^{\prime}=w_{i}, \quad\left(w^{\prime}, b_{i j}\right)=\zeta_{j}, \quad j \neq i .
$$

Тогда $w_{j}^{\prime}=2\left(\zeta_{j}-w_{j}\right)$ при $j \neq i$. Поэтому, учитывая неравенства $(19),(20)$, имеем

$$
\left|w^{\prime}-w\right| \leqslant 2 \sqrt{\sum_{j \neq i}\left|\zeta_{j}-\left(w_{i}+\frac{1}{2} w_{j}\right)\right|^{2}} \leqslant 2 x C \cdot(1+|w|)^{1-\frac{\gamma}{3}}, \quad C-\text { const. }
$$

Поскольку $w^{\prime}=\left(w_{1}^{\prime}, \ldots, w_{p}^{\prime}\right) \in \Sigma_{\zeta}^{i}$, то из последнего неравенства вытекает, что объединение шаров $D_{2 p}\left(w, y(1+|w|)^{1-\frac{\gamma}{3}}\right)$ при некотором $y>0$ покрьвает все пространство $\mathbb{C}^{p}$, когда $w$ пробегает плоскости $\Sigma_{\zeta}^{i}, i=1, \ldots, p, \zeta \in S^{p-1}$.

По построению оценка (17) выполняется на плоскостях $\Sigma_{\zeta}^{i}$, за исключением множества

$$
\bigcup_{f_{\zeta, i}(z)=0} D_{2 p}\left(z, d\left(1+\left|z_{0}\right|+|z|\right)^{\alpha}\right) .
$$

Увеличивая $|\alpha|, \alpha<0$, и уменьшая $d>0$, можно это исключительное множество сколь угодно уменьшить. Тем самым шары $D_{2 p}\left(w, y(1+|w|)^{1-\frac{\gamma}{3}}\right)$, когда $w$ пробегает $\Sigma_{\zeta}^{i}$ вне исключительных множеств, покрывают все пространство $\mathbb{C}^{p}$. Теорема 1 доказана.

\section{§ 2. Регуляризация плюрисубгармонических функций}

В этом параграфе доказывается теорема о приближении произвольной плюрисубгармонической функции посредством функций, удовлетворяющих дополнительным условиям.

Пусть $\beta(t) \in C_{0}^{\infty}(\mathbb{R}), \beta(t) \geqslant 0, \beta(t)=0$, когда $t \notin(0,1)$, и

$$
c_{p} \int \beta\left(t^{2}\right) t^{2 p-1} d t=1
$$

где $c_{p}$ - площадь единичной сферы в $\mathbb{C}^{p}$.

Через $L(u ; z, a)$ обозначим форму Леви функции $u$ в точке $z$ :

$$
L(u ; z, a)=\sum_{i, j=1}^{p} \frac{\partial^{2} u}{\partial z_{i} \partial \bar{z}_{j}} a_{i} \bar{a}_{j}
$$


ЛЕмма 7. Пусть $\rho(z)$ - положительная гладкая функиия и функиия и плюрисубгармонична в $\mathbb{C}^{p}$. Положим

$$
\tilde{u}(z)=\int u(z+\rho(z) \zeta) \beta\left(|\zeta|^{2}\right) d \lambda_{2 p}(\zeta)
$$

Тогда имеет место неравенство

$$
L(u ; z, a) \geqslant L(\ln \rho ; z, a) \frac{2 c_{p}(p-1)}{\rho(z)^{2 p-2}} \int_{0}^{\infty} \mu_{u}(z, \rho(z) t) t \beta\left(t^{2}\right) d t .
$$

Тем самым если $\ln \rho$ плюрисубгармонична в точке $z$, то функиия й также плюрисубгармонична в әтой точке.

ДокАЗАТЕльство. Зафиксируем точку $z_{0}=\left(z_{1}^{0}, z_{2}^{0}, \ldots, z_{p}^{0}\right) \in \mathbb{C}^{p}$ и определим функцию $\rho_{0}$ из равенства

$$
\begin{aligned}
\ln \rho_{0}(z)= & \ln \rho\left(z_{0}\right)+2 \sum_{m=1}^{p} \frac{\partial \ln \rho}{\partial z_{m}}\left(z_{0}\right)\left(z_{m}-z_{m}^{0}\right) \\
& +\sum_{m, n=1}^{p} \frac{\partial^{2} \ln \rho}{\partial z_{m} \partial z_{n}}\left(z_{0}\right)\left(z_{m}-z_{m}^{0}\right)\left(z_{m}-z_{m}^{0}\right) .
\end{aligned}
$$

По определению имеют место равенства

$$
\begin{aligned}
\rho\left(z_{0}\right) & =\rho_{0}\left(z_{0}\right), \\
\frac{\partial \ln \rho}{\partial z_{i}}\left(z_{0}\right) & =\frac{\partial \ln \left|\rho_{0}\right|}{\partial z_{i}}\left(z_{0}\right), \quad i=1, \ldots, p, \\
\frac{\partial^{2} \ln \rho}{\partial z_{i} \partial z_{j}}\left(z_{0}\right) & =\frac{\partial^{2} \ln \left|\rho_{0}\right|}{\partial z_{i} \partial z_{j}}\left(z_{0}\right), \quad i, j=1, \ldots, p .
\end{aligned}
$$

Определим вспомогательную функцию:

$$
u_{0}(z)=\int u\left(z+\rho_{0}(z) \zeta\right) \beta\left(|\zeta|^{2}\right) d \lambda(\zeta)
$$

Так как $\rho_{0}(z)$ голоморфна, то функция $u_{0}(z)$ плюрисубгармонична. Далее, применяя замену переменных $z+\rho_{0}(z) \zeta=w$, затем замену переменных $z+\left|\rho_{0}(z)\right| \zeta=w$, получим равенство

$$
\begin{aligned}
u_{0}(z) & =\int u(w) \beta\left(\left|\frac{z-w}{\rho_{0}(z)}\right|^{2}\right)\left|\rho_{0}(z)\right|^{-2 p} d \lambda_{2 p}(w) \\
& =\int u\left(z+\left|\rho_{0}(z)\right| \zeta\right) \beta\left(|\zeta|^{2}\right) d \lambda_{2 p}(\zeta) .
\end{aligned}
$$

Учитывая равенство первых производных функций $\ln \left|\rho_{0}\right|, \ln \rho$, нетрудно получить формулу

$$
\begin{aligned}
L\left(\tilde{u}-u_{0} ; z_{0}, a\right)= & L\left(\rho-\left|\rho_{0}\right| ; z_{0}, a\right) \\
& \times 2 \operatorname{Re} \int \sum_{m=1}^{p} \frac{\partial u\left(z_{0}+\rho\left(z_{0}\right) \zeta\right)}{\partial w_{m}} \zeta_{m} \beta\left(|\zeta|^{2}\right) d \lambda_{2 p}(\zeta) .
\end{aligned}
$$


Интегрируя по частям, последний интеграл можно записать в виде

$$
\begin{aligned}
& \int \sum_{m=1}^{p} \frac{\partial u\left(z_{0}+\rho\left(z_{0}\right) \zeta\right)}{\partial w_{m}} \zeta_{m} \beta\left(|\zeta|^{2}\right) d \lambda_{2 p}(\zeta) \\
& =\sum_{m=1}^{p} \frac{1}{\rho\left(z_{0}\right)} \int \frac{\partial u\left(z_{0}+\rho\left(z_{0}\right) \zeta\right)}{\partial \zeta_{m}} \zeta_{m} \beta\left(|\zeta|^{2}\right) d \lambda_{2 p}(\zeta) \\
& =-\frac{1}{\rho\left(z_{0}\right)} \int u\left(z_{0}+\rho\left(z_{0}\right) \zeta\right)\left(p \beta\left(|\zeta|^{2}\right)+|\zeta|^{2} \beta^{\prime}\left(|\zeta|^{2}\right)\right) d \lambda_{2 p}(\zeta) .
\end{aligned}
$$

Воспользуемся формулой Привалова. Тогда

$$
\begin{aligned}
& \int u\left(z_{0}+\rho\left(z_{0}\right) \zeta\right)\left(p \beta\left(|\zeta|^{2}\right)+|\zeta|^{2} \beta^{\prime}\left(|\zeta|^{2}\right)\right) d \lambda_{2 p}(\zeta) \\
& \quad=c_{p} \int_{0}^{\infty}\left(\int_{0}^{\rho\left(z_{0}\right) t} \frac{\mu_{u}\left(z_{0}, \tau\right)}{\tau^{2 p-1}}(2 p-2) d \tau\right)\left(p \beta\left(t^{2}\right)+t^{2} \beta^{\prime}\left(t^{2}\right)\right) t^{2 p-1} d t
\end{aligned}
$$

Отсюда, интегрируя по частям, получим

$$
\begin{gathered}
\int u\left(z_{0}+\rho\left(z_{0}\right) \zeta\right)\left(p \beta\left(|\zeta|^{2}\right)+|\zeta|^{2} \beta^{\prime}\left(|\zeta|^{2}\right)\right) d \lambda_{2 p}(\zeta) \\
=-c_{p}(p-1) \int_{0}^{\infty} t^{2 p} \beta\left(t^{2}\right) \frac{\mu_{u}\left(z_{0}, \rho\left(z_{0}\right) t\right)}{\rho\left(z_{0}\right)^{2 p-2} t^{2 p-1}} d t \\
=-c_{p}(p-1) \int_{0}^{\infty} \frac{\mu_{u}\left(z_{0}, \rho\left(z_{0}\right) t\right)}{\rho\left(z_{0}\right)^{2 p-2}} t \beta\left(t^{2}\right) d t .
\end{gathered}
$$

Подставляя это выражение в (22), затем в (21), получим формулу

$$
L\left(\tilde{u}-u_{0} ; z_{0}, a\right)=L\left(\rho-\left|\rho_{0}\right| ; z_{0}, a\right) \frac{2 c_{p}(p-1)}{\rho\left(z_{0}\right)^{2 p-1}} \int_{0}^{\infty} \mu_{u}\left(z_{0}, \rho\left(z_{0}\right) t\right) t \beta\left(t^{2}\right) d t .
$$

По определению функции $\rho_{0}$ имеем

$$
L\left(\rho-\left|\rho_{0}\right| ; z_{0}, a\right)=L\left(\ln \rho ; z_{0}, a\right) \rho\left(z_{0}\right) .
$$

Таким образом, доказана формула

$$
L\left(\tilde{u} ; z_{0}, a\right)=L\left(u_{0} ; z_{0}, a\right)+L\left(\ln \rho ; z_{0}, a\right) \frac{2 c_{p}(p-1)}{\rho\left(z_{0}\right)^{2 p-2}} \int_{0}^{\infty} \mu_{u}\left(z_{0}, \rho\left(z_{0}\right) t\right) t \beta\left(t^{2}\right) d t
$$

из которой следует утверждение леммы 7.

Для дальнейшего нам потребуется оценка интеграла от модуля плюрисубгармонической функции. При этом будет использовано следуюшее утверждение из работы [10] (см. также [11]). 
Лемма 8. Существует постоянная $M_{m}$ такая, что для любой функиии $q(x) \in K_{\rho}$ и для любой субгармонической функции и в $\mathbb{R}^{m}$, удовлетворяющей условиям

$$
\begin{aligned}
& u(0)=0, \\
& u(x) \leqslant q(|x|), \quad x \in \mathbb{R}^{m},
\end{aligned}
$$

имеют место неравенства

$$
\int_{B}|u(x)| d \lambda_{m}(x) \leqslant M_{m}\left(\lambda_{m}(B)\right)^{\frac{2}{m}} 4^{\rho} q\left(\max _{x \in B}|x|\right)\left(\max _{x \in B}|x|\right)^{m-2}
$$

nрu $m>2 u$

$$
\int_{B}|u(x)| d \lambda(x) \leqslant M_{2} 4^{\rho} \lambda(B) q\left(\max _{z \in B}|x|\right) \ln \frac{16 \pi(\max |x|)^{2}}{e \lambda(B)}
$$

npu $m=2$.

Лемма 9. Существуют постоянные $A_{p}, B_{p}>1$ такие, что для любой функции $q(x) \in K_{\rho}$ и для любой плюрисубгармонической функции $\varphi$ в $\mathbb{C}^{p}$, удовлетворяющей условиям

$$
\begin{aligned}
& \varphi(0)=0, \\
& \varphi(z) \leqslant q(|z|), \quad z \in \mathbb{C}^{p},
\end{aligned}
$$

имеет место неравенство

$$
\int_{D^{p}(w, R)}|\varphi(z)| d \lambda_{2 p}(z) \leqslant A_{p} B_{p}^{\rho} q(T) R^{2 p} \ln ^{p} \frac{T+1}{R_{0}} e,
$$

где $T=|w|+R, \quad R_{0}=\min (1, R), \quad w=\left(w_{1}, \ldots, w_{p}\right) \in \mathbb{C}^{p}, D^{p}(w, R)$ - поликруг $D\left(w_{1}, R\right) \times \cdots \times D\left(w_{p}, R\right)$.

ДоКАЗАТЕЛЬСТВо проведем по индукции по размерности пространства $\mathbb{C}^{p}$. Для $p=1$ утверждение леммы вытекает из леммы 8. Пусть лемма верна в $\mathbb{C}^{p-1}$. Для точки $z=\left(z_{1}, \ldots, z_{p}\right) \in \mathbb{C}^{p}$ через $z^{\prime}$ обозначим точку $\left(z_{2}, \ldots, z_{p}\right) \in \mathbb{C}^{p-1}$. По условиям леммы функция

$$
\psi(\zeta)=\varphi(\zeta, 0, \ldots, 0), \quad \zeta \in \mathbb{C},
$$

обладает свойствами

$$
\begin{aligned}
& \psi(0)=0 \\
& \psi(\zeta) \leqslant q(|\zeta|), \quad \zeta \in \mathbb{C} .
\end{aligned}
$$

Положим

$$
\begin{aligned}
& \beta=q(0)\left(\frac{T+1}{R_{0}} e\right)^{\alpha}, \\
& s=s(\zeta)=\left(\frac{T+1}{R_{0}} e\right)^{-\alpha-1} 3|\zeta|,
\end{aligned}
$$


где $\alpha=\rho+2 p^{2}, \quad R_{0}=\min (1, R)$. По лемме 3 в точках $\zeta \in \mathbb{C}$, являюшихся $(\beta, s)$-нормальными по мере $\mu_{\psi}$, имеет место оценка снизу

$$
\psi(\zeta) \geqslant-A B^{\rho} q(|\zeta|) \ln \left(\frac{T+1}{R_{0}} e\right)^{\alpha+1}-2 q(0)
$$

Так как $q(0) \leqslant q(|\zeta|)$, то для некоторых постоянных $A, B \geqslant 1$ выполняется неравенство

$$
\psi(\zeta) \geqslant-A B^{\rho} q(|\zeta|) \ln \frac{T+1}{R_{0}} e .
$$

Множество точек $\zeta \in D\left(w_{1}, R\right)$, не являюшихся $(\beta, s(\zeta))$-нормальными по мере $\mu_{\psi}$, обозначим через $E_{0}$. Для $\zeta \notin E_{0}$ рассмотрим функцию

$$
\varphi_{\zeta}\left(z^{\prime}\right)=\varphi\left(\zeta, z^{\prime}\right)-\psi(\zeta), \quad z^{\prime} \in \mathbb{C}^{p-1} .
$$

Эта функция плюрисубгармонична в $\mathbb{C}^{p-1}$, и если $\zeta \notin E_{0}$, то

$$
\begin{aligned}
\varphi_{\zeta}\left(z^{\prime}\right) & \leqslant q\left(|\zeta|+\left|z^{\prime}\right|\right)+A B^{\rho} q(|\zeta|) \ln \left(\frac{T+1}{R_{0}} e\right) \\
& \leqslant A B^{\rho} q\left(|\zeta|+\left|z^{\prime}\right|\right) \ln \left(\frac{T+1}{R_{0}} e\right) .
\end{aligned}
$$

Функция

$$
q_{1}(x)=A B^{\rho} q(|\zeta|+x) \ln \frac{(T+1) e}{R_{0}},
$$

как нетрудно проверить, принадлежит классу $K_{\rho}$. По допушению индукции имеет место оценка

$$
\int_{D^{p-1}\left(w^{\prime}, R\right)}\left|\varphi_{\zeta}\left(z^{\prime}\right)\right| d \lambda_{2(p-1)}\left(z^{\prime}\right) \leqslant A_{p-1} B_{p-1}^{\rho} q_{1}\left(T^{\prime}\right) R^{2(p-1)} \ln ^{p-1} \frac{\left(T^{\prime}+1\right) e}{R_{0}},
$$

где $T^{\prime}=\left|w^{\prime}\right|+R<T$. Отсюда, учитьвая определение функции $q_{1} \in K_{\rho}$, получим

$$
\int_{D^{p-1}\left(w^{\prime}, R\right)}\left|\varphi_{\zeta}\left(z^{\prime}\right)\right| d \lambda_{2(p-1)}\left(z^{\prime}\right) \leqslant A B^{\rho} q(T) R^{2(p-1)} \ln ^{p} \frac{(T+1) e}{R_{0}} .
$$

Положим

$$
E=\left\{z=\left(z_{1}, \ldots, z_{p}\right) \in D^{p}(w, R): z_{1} \in E_{0}\right\} .
$$

Тогда из последнего неравенства вытекает оценка

$$
\int_{D^{p}(w, R) \backslash E}|\varphi(z)| d \lambda_{2 p}(z) \leqslant \pi A B^{\rho} q(T) R^{2 p} \ln ^{p} \frac{(T+1) e}{R_{0}} .
$$

Оценим $\lambda_{2 p}(E)$. По определению $E_{0}$ для любой точки $\zeta \in E_{0}$ найдется число $r \leqslant s<1$ такое, что

$$
\mu_{\psi}(\zeta, r) \geqslant \beta r
$$


По лемме Хеймана [9] существует счетная система точек $\zeta_{j}$ такая, что

$$
E_{0} \subset \bigcup D\left(\zeta_{j}, r_{j}\right), \quad \mu_{\psi}\left(\zeta_{j}, r_{j}\right) \geqslant \beta r_{j}
$$

причем каждая точка плоскости попадает не более чем в шесть кругов $D\left(\zeta_{j}, r_{j}\right)$. Очевидно, справедливы неравенства

$$
\beta \sum_{j} r_{j} \leqslant \sum_{j} \mu_{\psi}\left(\zeta_{j}, r_{j}\right) \leqslant 6 \mu_{\psi}\left(w_{1}, R+1\right) \leqslant 6 \mu_{\psi}(0, T+1) .
$$

Из формулы Привалова и из условий леммы следует, что

$$
\mu_{\psi}(0, T+1) \leqslant e^{\rho} q(T+1) \leqslant e^{\rho} q(1)(T+1)^{\rho} .
$$

Отсюда получим оценки

$$
\sum_{j} r_{j} \leqslant 6 e^{\rho} \frac{q(1)}{q(0)}(T+1)^{\rho}\left(\frac{T+1}{R_{0}} e\right)^{-\alpha} .
$$

Учитывая, что $r_{j}<1$ и $q(1) \leqslant 2^{\rho} q(0)$, имеем неравенство

$$
\lambda_{2}\left(E_{0}\right) \leqslant A B^{\rho}(T+1)^{\rho}\left(\frac{T+1}{R_{0}} e\right)^{-\alpha} .
$$

Поскольку $E \subset E_{0} \times D^{p-1}\left(w^{\prime}, R\right)$, то

$$
\lambda_{2 p}(E) \leqslant A B^{\rho}(T+1)^{\rho} R^{2 p-2}\left(\frac{T+1}{R_{0}} e\right)^{-\alpha} .
$$

Отсюда и из леммы 8 получим оценку

$$
\begin{aligned}
\int_{E}|\varphi(z)| d \lambda_{2 p}(z) & \leqslant A B^{\rho}(T+1)^{\frac{\rho}{p}} R^{2-\frac{2}{p}}\left(\frac{T+1}{R_{0}} e\right)^{-\frac{\alpha}{p}} q(T) T^{2 p-2} \\
& \leqslant A B^{\rho} q(T) R^{2 p}(T+1)^{\frac{\rho}{p}+2 p-2} R^{2-\frac{2}{p}-2 p}\left(\frac{T+1}{R_{0}} e\right)^{-\frac{\alpha}{p}}
\end{aligned}
$$

По определению чисел $\alpha$ и $R_{0}$ окончательно получим

$$
\int_{E}|\varphi(z)| d \lambda_{2 p}(z) \leqslant A B^{\rho} q(T) R^{2 p}
$$

Это неравенство вместе с (23) дает утверждение леммы 9. 
ТЕОРема 2. Для любой плюрисубгармонической функиии ч, удовлетворяющей условию

$$
\varphi(z) \leqslant C \cdot(1+|z|)^{\rho}, \quad z \in \mathbb{C}^{p}, \quad C-\text { const }
$$

существует гладкая плюрисубгармоническая функиия $\widetilde{\varphi}$, которая вне некоторого множества Е удовлетворяет асимптотическому соотношению

$$
|\varphi(z)-\widetilde{\varphi}(z)| \leqslant C \cdot(1+|z|)^{\rho+\gamma-1} \ln (|z|+e)
$$

где $\gamma=\max \left(1-\frac{\rho}{5}, 0\right), C$ - const. Кроме того, функиия $\widetilde{\varphi}$ обладает свойством

$$
|\widetilde{\varphi}(z)-\widetilde{\varphi}(w)| \leqslant(1+|z|)^{\rho-\gamma} \ln ^{p}(|z|+e)|z-w|
$$

если $|z-w| \leqslant 1$. Множество Е может быть покрыто системой шаров $D_{2 p}\left(z_{j}, r_{j}\right) \operatorname{mak}$, чmo

$$
\sum_{\frac{R}{2} \leqslant\left|z_{j}\right| \leqslant R} r_{j}^{2 p-1}=O\left(\frac{R^{2 p-1}}{\ln (R+e)}\right), \quad R \rightarrow \infty .
$$

ДокАЗАтЕльство. Пусть функция $\beta(t) \geqslant 0$ (равна 0 при $t \notin(0,1))$ бесконечно дифференцируема и

$$
c_{p} \int_{0}^{\infty} \beta\left(t^{2}\right) t^{2 p-1} d t=1
$$

Положим

$$
\widetilde{\varphi}(z)=\int \varphi\left(z+(1+|z|)^{\gamma} \zeta\right) \beta\left(|\zeta|^{2}\right) d \lambda_{2 p}(\zeta)
$$

где $\gamma=\max \left(1-\frac{\rho}{5}, 0\right)$. При фиксированном $z$ через $R$ обозначим $(1+|z|)^{\gamma}$. По формуле Привалова имеем

$$
\widetilde{\varphi}(z)-\varphi(z)=c_{p} d_{p} \int\left(\int_{0}^{R t} \frac{\mu_{\varphi}(z, \tau)}{\tau^{2 p-1}} d \tau\right) \beta\left(t^{2}\right) t^{2 p-1} d t
$$

где $d_{p}=2(p-1), p>1, d_{1}=1$ и $c_{p}-$ площадь поверхности единичной сферы в $\mathbb{C}^{p}$.

Пусть $E-$ множество в $\mathbb{C}^{p}$ такое, что для любой точки $z \notin E$ выполняется неравенство

$$
\mu_{\varphi}(z, \tau) \leqslant(1+|z|)^{\rho-1} \ln (|z|+e) \tau^{2 p-1}, \quad \tau \in\left(0, \frac{|z|}{2}\right)
$$

Тогда для любой точки $z \notin E$ имеем оценку

$$
0 \leqslant \widetilde{\varphi}(z)-\varphi(z) \leqslant d_{p}(1+|z|)^{\rho+\gamma-1} \ln (|z|+e)
$$


Докажем оценку (26). Прямым вычислением получим, что имеет место неравенство

$$
\left|\operatorname{grad}_{z}\left[\beta\left(\frac{|\zeta-z|^{2}}{(1+|z|)^{2 \gamma}}\right)(1+|z|)^{-2 p \gamma}\right]\right| \leqslant C \cdot(1+|z|)^{-(2 p+1) \gamma},
$$

причем константа $C$ не зависит от $\zeta$. Поэтому из леммы 9 вытекает оценка

$$
\begin{aligned}
|\operatorname{grad} \widetilde{\varphi}(z)| & \leqslant C \cdot(1+|z|)^{-(2 p+1) \gamma} \int_{D^{p}(z, R)}|\varphi(\zeta)| d \lambda_{2 p}(\zeta) \\
& \leqslant C \cdot(1+|z|)^{p-\gamma} \ln ^{p}(|z|+e) .
\end{aligned}
$$

Отсюда следует соотношение (24). Получим оценки множества $E$. В силу $(26)$ для любой точки $z \in E$ найдется $\tau=\tau(z)<\frac{|z|}{2}$ такое, что

$$
\mu_{\varphi}(z, \tau) \geqslant(1+|z|)^{\rho-1} \ln (|z|+e) \tau^{2 p-1} .
$$

По лемме Хеймана [9] сушествует не более чем счетное множество точек $z_{j} \in E$ такое, что система шаров $D_{2 p}\left(z_{j}, \tau_{j}\right), \tau_{j}=\tau\left(z_{j}\right)$, покрывает все множество $E$, причем каждая точка пространства попадает не более чем в $k_{p}$ шаров из этой системы. Очевидно, выполняется неравенство

$$
\sum_{\frac{R}{2} \leqslant\left|z_{j}\right| \leqslant R} \tau_{j}^{2 p-1}\left(1+\left|z_{j}\right|\right)^{\rho-1} \ln \left(\left|z_{j}\right|+e\right) \leqslant k_{p} \mu_{\varphi}(0,2 R) .
$$

Тогда

$$
\sum_{\frac{R}{2} \leqslant\left|z_{j}\right| \leqslant R} \tau_{j}^{2 p-1} \leqslant C \frac{R^{2 p-1}}{\ln (R+e)}, \quad C-\text { const. }
$$

Тем самым теорема 2 доказана.

Комбинируя теоремы 1 и 2 , получим следующее утверждение.

Теорема 3. Для любой плюрисубгармонической функции ч в $\mathbb{C}^{p}$ порядка роста $\rho$ и для любого $\epsilon>0$ существует иелая функиия $f$, для которой вне некоторого множества $E$, удовлетворяющего соотношению (25), выполнено неравенство

$$
|\varphi(z)-\ln | f(z)|| \leqslant C \cdot(1+|z|)^{\alpha+\epsilon}
$$

əде $\alpha=\max \left(\frac{4}{5} \rho, \rho-1\right), C$ - const.

В заключение приведем теорему, являющуюся непосредственным следствием теоремы 1.

Teоpema 4. Пусть $K$ - компакт в $D_{2 p}(0,1) u$

$$
H_{K}(z)=\max _{\lambda \in K} \operatorname{Re}(\lambda, z)
$$

- опорная функция этого компакта.

Тогда существует иелая функиия $F(z)$, которая вне множества

$$
S_{F}(\alpha, d)=\bigcup_{F(z)=0} D\left(z, d(1+|z|)^{\alpha}\right)
$$

удовлетворяет соотношенил

$$
|\ln | f(z)\left|-H_{K}(z)\right| \leqslant C \cdot(1+|z|)^{\frac{2}{3}},
$$

әде константа $C$ зависит от $\alpha, d, d>0$, и не зависит от $K$. 
ДокАЗАТЕЛЬСтво. Пусть $z, w \in \mathbb{C}^{p}$ и $H_{K}(z)=\operatorname{Re}(\lambda, z)$. Тогда, очевидно,

$$
H_{K}(z)-H_{K}(w) \leqslant \operatorname{Re}(\lambda, z-w) \leqslant|\lambda||z-w| .
$$

Поэтому имеет место неравенство

$$
\left|H_{K}(z)-H_{K}(w)\right| \leqslant \max _{\lambda \in K}|\lambda||z-w|,
$$

из которого по теореме 1 следует утверждение теоремы 3.

\section{Список литературы}

1. Азария В. С. О лучах вполне регулярного роста целой функции // Матем. сб. 1969. T. 79. № 4. C. 463-476.

2. Азарин В. C. Об асимптотическом поведении субгармонических функций конечного порядка // Матем. сб. 1979. Т. 108(150). С. 147-167.

3. Юлмухаметов Р. С. Приближение субгармонических функций // Матем. сб. 1984. T. 124. № 3. C. 393-416.

4. Юлмухаметов Р. С. Аппроксимация субгармонических функций // Analysis Mathematica. 1985 . T. 11. № 3. C. 257-282.

5. Любарский Ю. И., Содин М. Л. Аналог функции типа синуса для выпуклых областей. Препринт ФТИНТ АН УССР. Т. 17, 1986.

6. Sigurdsson R. Growth properties of analytic and plurisubharmonic functions of finite order. Lund university, 1984.

7. Юлмухаметов Р. С. Асимптотика плюрисубгармонических функций. Препринт БФАН CCCP, 1986.

8. Хермандер П. Введение в теорию функций нескольких комплексных переменных. М.: Мир, 1968.

9. Ланджоф Н. С. Основы современной теории потенциала. М.: Наука, 1966.

10. Фаворов С. Ю. О сложении индикаторов целых и субгармонических функций // Матем. сб. 1978. Т. 105. №1. С. 128-139.

11. Азарин В. С. Об одном характеристическом свойстве функций вполне регулярного роста внутри угла // Теория функций, функциональный анализ и их приложения. Харьков, 1966. T. 2. C. $55-66$.

12. Левин Б. Я. Целые функции. М.: Изд-во МГУ, 1971.

Башкирский государственный университет

Поступило в редакцию 